(C) 2008 The Japan Society of Applied Physics

\title{
Formation Mechanism of 100-nm-Scale Periodic Structures in Silicon Using Magnetic-Field-Assisted Anodization
}

\author{
Daihei HIPPO ${ }^{1,5 *}$, Yoshifumi NAKAmine ${ }^{1}$, Kei URAKawA ${ }^{2}$, Yoshishige TsuchiYA ${ }^{1,3,5}$, \\ Hiroshi Mizuta ${ }^{3,4,5}$, Nobuyoshi KoshidA ${ }^{2,5}$, and Shunri ODA ${ }^{1,5}$ \\ ${ }^{1}$ Quantum Nanoelectronics Research Center, Tokyo Institute of Technology, 2-12-1 O-okayama, Meguro-ku, Tokyo 152-8552, Japan \\ ${ }^{2}$ Graduate School of Engineering, Tokyo University of Agriculture and Technology, Koganei, Tokyo 184-8588, Japan \\ ${ }^{3}$ School of Electronics and Computer Science, University of Southampton, Highfield, Southampton SO17 1BJ, U.K. \\ ${ }^{4}$ Department of Physical Electronics, Tokyo Institute of Technology, 2-12-1 O-okayama, Meguro-ku, Tokyo 152-8552, Japan \\ ${ }^{5}$ SORST-JST (Japan Science and Technology Agency), Kawaguchi, Saitama 332-0012, Japan
}

(Received December 3, 2007; revised April 16, 2008; accepted May 8, 2008; published online September 12, 2008)

We demonstrate highly directional etching in silicon $100 \mathrm{~nm}$ in diameter with an aspect ratio of 160 with no spiking on the pore walls using magnetic-field-assisted anodization. The relationship between the surface geometry of a silicon electrode and its highly directional etching properties have been investigated. Specifically, we show that the pore shape and pore wall orientation are not determined by the surface pattern but by the etching mechanisms specific to the magnetic-field-assisted anodization. These etching mechanisms enable highly directional and high aspect ratio etching at diameters below $100 \mathrm{~nm}$ in scale. [DOI: 10.1143/JJAP.47.7398]

KEYWORDS: silicon nanostructures, magnetic-field-assisted anodization, mesoporous silicon, pattern transfer

\section{Introduction}

Electrochemical etching is a powerful tool for producing patterned structures without the use of standard lithographic techniques. ${ }^{1-3)}$ The applications of electrochemical etching vary from photonic crystals ${ }^{4,5)}$ and sensors ${ }^{6)}$ to massively parallel Brownian ratchets. ${ }^{7)}$ To date, patterned structures with high aspect ratios and highly uniform trenches produced by electrochemical etching methods are used only for macroporous silicon with pore diameters above $300 \mathrm{~nm} .^{8,9)}$ However, a few reports have appeared on studies of uniform pores below $200 \mathrm{~nm}$ in diameter. ${ }^{10,11)}$ In the formation mechanism for macroporous silicon, diffusion lengths of minority carriers play a key role, which can be controlled by homogeneous backside illumination. ${ }^{1,2,12)}$ The lower limit for the pore diameter is established by breakdown, which leads to light-independent pore growth and spiking. ${ }^{13)}$ On the other hand, the mesoporous silicon formation mechanism depends on the space charge region around pores which can be controlled by the doping density of the silicon substrate. ${ }^{14)}$ In particular, highly doped silicon substrates are required to achieve pore geometries below $100 \mathrm{~nm}$ in scale, which then enables a variety of applications including very large scale integration (VLSI), micro-electromechanical systems (MEMS), and three-dimensional (3D) photonic crystals with higher frequencies. ${ }^{15)}$ However, space charge regions around pores experience greater fluctuations as the doping density increases, and mesopore growth processes are greatly affected by local electric field distributions. These effects result in spiking or bending of pores, and they are the major problems with the existing mesopore formation techniques. The controlled anodization technique using an external magnetic field has been applied to mesopore formation and resulted in improved etching properties on the 100 -nm-scale. ${ }^{16)}$ In this technique an external magnetic field is applied to control the motion of holes, which initiate the electrochemical dissolution of silicon. In this paper, we report the mechanism of onedirectional etching in silicon using this method.

*E-mail address: d-hippo@neo.pe.titech.ac.jp

\section{Experimental Methods}

We fabricated samples with two different types of surface geometries: rectangular-parallelpiped etchpits (Sample A), and inverse-pyramidal etchpits (Sample B). Both samples were prepared using heavily doped $(0.04-0.06 \Omega \cdot \mathrm{cm})$ n-type $\operatorname{Si}(100)$ wafers.

\subsection{Rectangular-parallelpiped etchpits (Sample A)}

Prior to anodization, an etching process in silicon, the sample surface is pre-patterned by electron beam (EB) lithography, after which electron cyclotron resonance reactive ion etching (ECR-RIE) is performed on the sample surface for $7 \mathrm{~min}$ to form shallow etchpits on the silicon substrate. The etching gas is $\mathrm{CF}_{4}$, and the chamber pressure during the etching process is at $1.0 \times 10^{-4}$ Torr. Anodization is performed in the dark in dilute $\mathrm{HF}(10 \%)$ solution at a constant current density of $12 \mathrm{~mA} / \mathrm{cm}^{2}$ for $10 \mathrm{~min}$. The temperature of the solution is kept at $0{ }^{\circ} \mathrm{C}$. The anodization is performed with an external magnetic field applied to the $\mathrm{Si}$ substrate perpendicular to the surface. The magnitude of the applied magnetic field is $1.9 \mathrm{~T}$.

\subsection{Inverse-pyramidal etchpits (Sample B)}

Prior to the EB lithography process, the substrate is thermally oxidized at $1000^{\circ} \mathrm{C}$ for $1 \mathrm{~h}$. Afterwards, EB lithography and ECR-RIE are performed under the same conditions as described for Sample A. Before the anodization process, the samples were immersed in $20 \% \mathrm{KOH}$ solution $\left(\mathrm{KOH}: \mathrm{H}_{2} \mathrm{O}=1: 4\right)$ at $80^{\circ} \mathrm{C}$ for $1 \mathrm{~min}$ to form inverse-pyramidal etchpits. The anodization is then performed under the same conditions as described for Sample A.

\section{Results}

\subsection{Rectangular-parallelpiped etchpits (Sample A)}

Figure 1(a) shows the cross-sectional scanning electron microscope (SEM) image of Sample A after anodization was performed. Straight vertical etching with a pore diameter of $80 \mathrm{~nm}$ was realized, although the pores seem intermittent in this picture. This is partly due to the difficulties of cleaving 


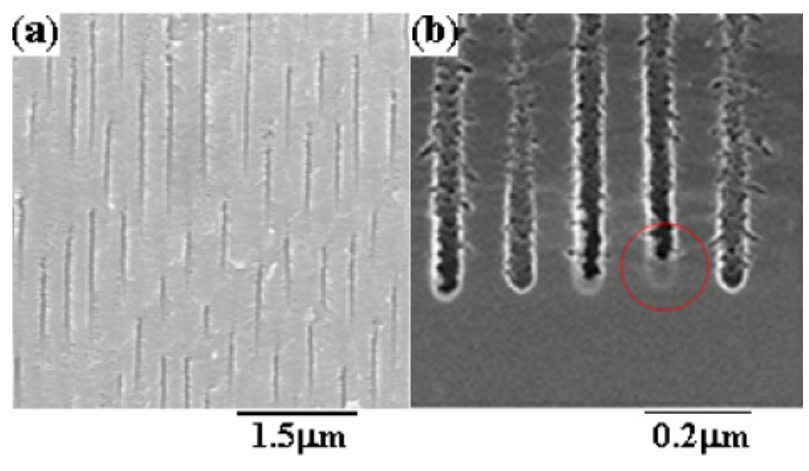

Fig. 1. (Color online) Cross-sectional SEM image of Sample A after anodization (a) in the middle and (b) at the bottom of pores.

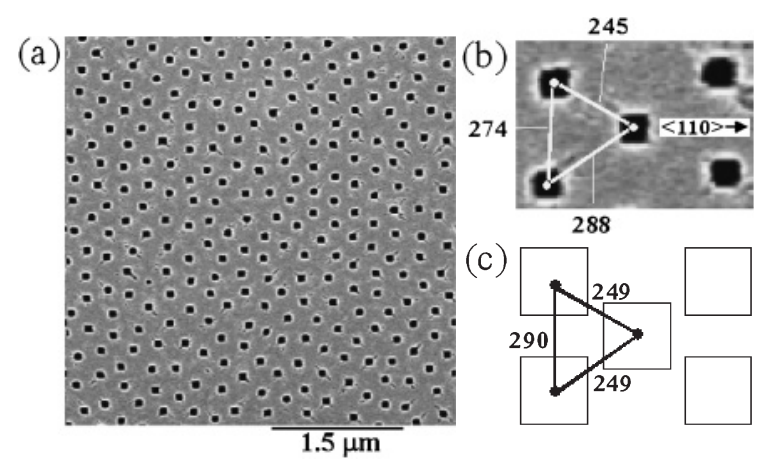

Fig. 3. (a) Surface SEM image of Sample A after removal of initial $10 \mu \mathrm{m}$ layer. (b) Magnification of a single unit of the fabricated structures, and (c) the unit of EB pattern initially formed on the sample surface. The numbers indicate corresponding pore distances in units of $\mathrm{nm}$.
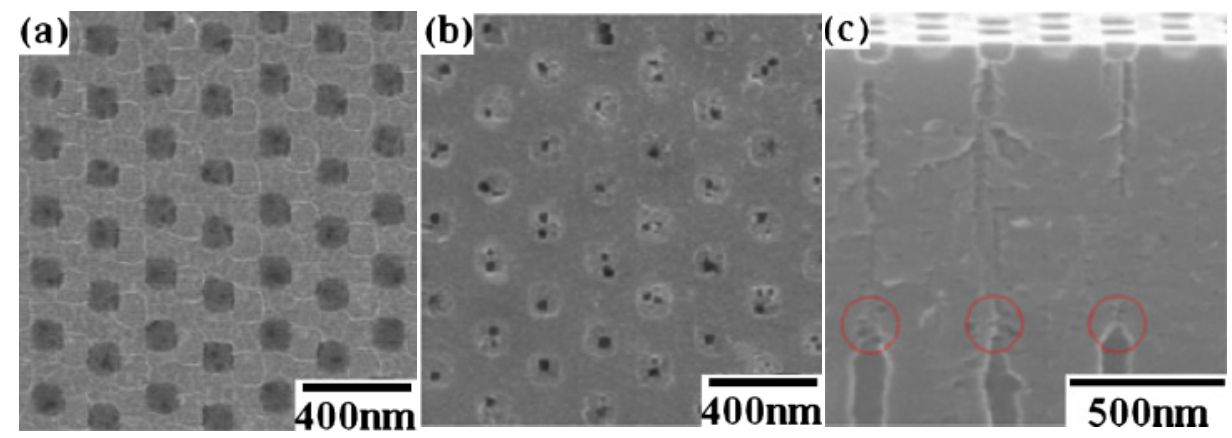

Fig. 2. (Color online) Surface SEM image of Sample A (a) before and (b) after the EB resist was removed. (c) Cross-sectional SEM image near the sample surface.

the sample with an accuracy below $80 \mathrm{~nm}$ and a possibility that the pore growth direction fluctuates. Figure 1(b) shows the bottom of pores which indicates that the pore tips are hemispherical. It should be noted that oxide formation is observed not only at pore tips, but at pore walls as well, as indicated in the circle in the figure. Figures 2(a) and 2(b) show the surface SEM images of Sample A before and after the EB resist (ZEP520) is removed, respectively. As can be seen from these images, the starting points of the dissolution process consist of one or more corners of the depressions, some of which are connected. Figure 2(c) shows the crosssectional SEM image of Sample A near the surface. Initially, pores grow with small diameters and they enlarge at a certain depth as indicated in the circles in Fig. 2(c). Next, the sample surface is mechanically polished to remove the first $10 \mu \mathrm{m}$ layer of the surface to investigate how the initial pattern on the top surface is projected into deeper regions. Figure 3(a) shows the SEM image of the surface of Sample A after removal of the first $10 \mu \mathrm{m}$ layer, and Fig. 3(b) shows the magnification of a single unit of the fabricated structures. The numbers indicate corresponding pore distances in units of $\mathrm{nm}$. Figure 3(c) shows the unit of the EB pattern formed on the sample surface. The geometry of the pores is congruent with the initial pattern on the top surface, although slight deviations from the designed pattern cannot be avoided. To evaluate the magnitude of pore pitch distribution, we measured pore distances for 500 points and plotted the data in a histogram. Figure 4(a) shows the histogram of pore distances in the sample fabricated under the same conditions as described for Sample A without an

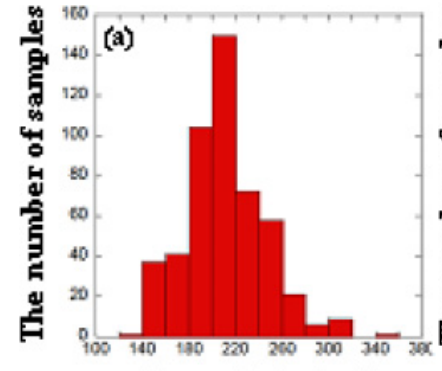

Pore pitch (nm)

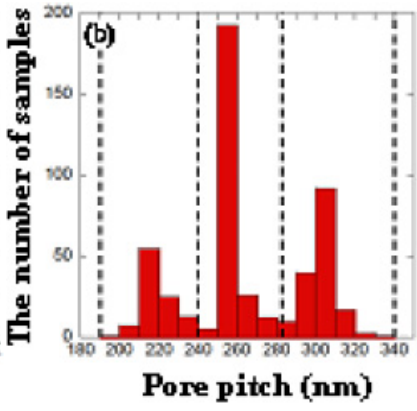

Fig. 4. (Color online) The histograms of pore distances. (a) The sample fabricated under the same conditions as Sample A without an EB pattern on the top surface, and (b) Sample A with an EB pattern on the top surface.

EB pattern on the top surface. Only one peak is centered around $220 \mathrm{~nm}$. Figure 4(b) shows the histogram of pore distances in Sample A, which has an EB pattern on the top surface. Two main peaks appear at 290 and $250 \mathrm{~nm}$ and one minor peak is at $220 \mathrm{~nm}$. The former two peaks correspond to the pore distances of the initial EB pattern on the top surface, as shown in Fig. 3(c), and the ratio of these two peaks is 2 to 1, which is consistent with the designed EB parameters. It should be noted that pore formation occurs even without a surface pattern, and the pore distances are mainly determined by the concentration of dopant in the silicon substrate. Equation (1) relates the width of the space charge region to the applied voltage, assuming a planar onedimensional Schottky contact ${ }^{17)}$ 

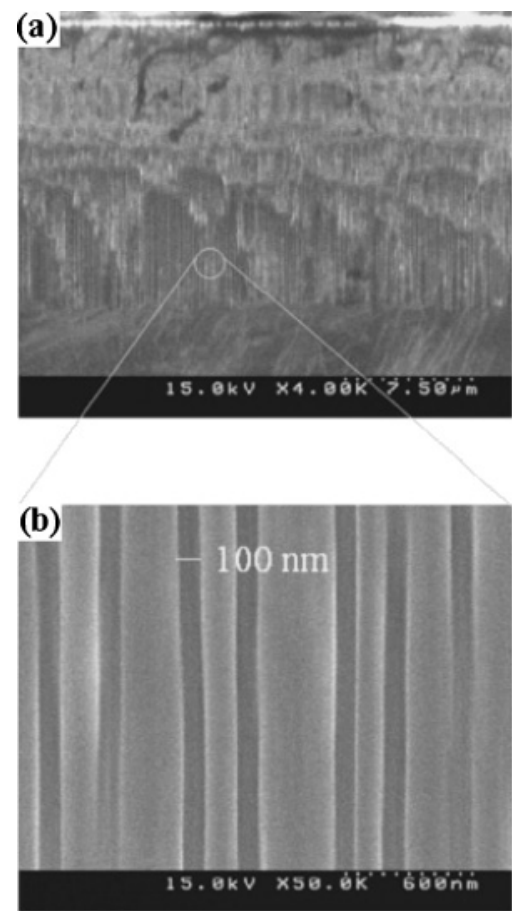

Fig. 5. Cross-sectional SEM images of Sample B after anodization is performed: (a) the whole structure, and (b) a magnification of the bottom part of the pores.

$$
l_{\mathrm{SCR}}=\sqrt{\frac{2 \varepsilon_{0} \varepsilon_{\mathrm{Si}} U_{\mathrm{SCR}}}{e N_{\mathrm{D}}}},
$$

where $\varepsilon_{0}$ is the dielectric constant of vacuum, $\varepsilon_{\mathrm{Si}}$ is the dielectric constant of silicon, $U_{\mathrm{SCR}}$ is the applied voltage, $e$ is the elementary charge, and $N_{\mathrm{D}}$ is the concentration of dopants. Comparing Figs. 4(a) and 4(b), the small peak observed at $220 \mathrm{~nm}$ in Fig. 4(b) is assumed to originate from naturally formed pores determined by the doping concentration of the silicon substrate as indicated in eq. (1).

\subsection{Inverse-pyramidal etchpits (Sample B)}

Figure 5(a) shows the cross-sectional SEM image of Sample B after anodization is performed. Figure 5(b) shows the magnification of the bottom part of the pores. The depth of the pores is approximately $16 \mu \mathrm{m}$ and the pore diameter is $100 \mathrm{~nm}$; thus the aspect ratio of the pores is estimated to be 160. The most prominent feature in this picture is the flatness of the pore walls. For reference, another sample was fabricated in the same conditions as described for Sample B, changing only the distances between the pores in the surface patterns. Figure 6 shows cross-sectional SEM images of the samples after anodization is performed. In the case of the sample with $850 \mathrm{~nm}$ pore distances, branching of pores is clearly observed in Fig. 6(a). On the other hand, no branching of pores is observed for the sample with $1000 \mathrm{~nm}$ pore distances as shown in Fig. 6(b). Both samples are anodized at the same current density, and therefore it is suggested that the quality of pore walls depends on the current per pore tip.

\section{Discussion}

\subsection{Overall reaction mechanisms}

In acidic electrolytes containing fluoride, silicon is stable at open circuit potentials, while electrochemical dissolution
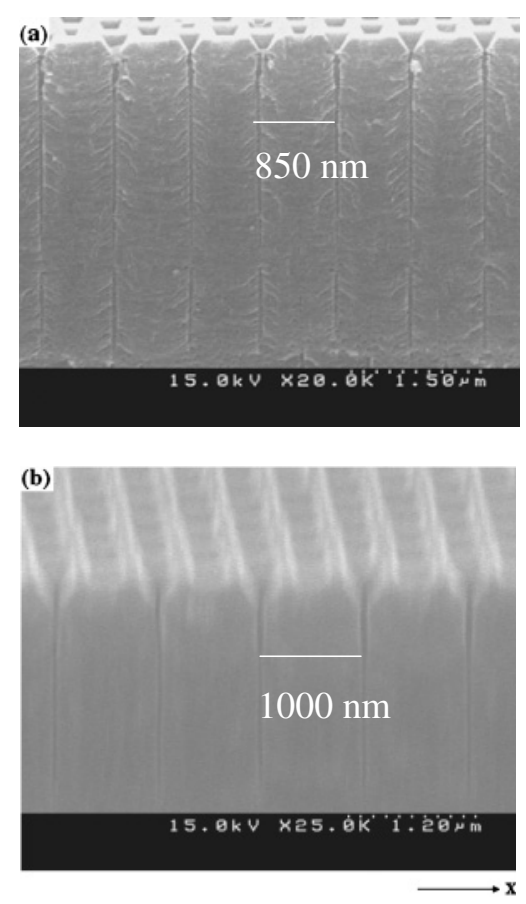

Fig. 6. Cross-sectional SEM images of the samples after anodization is performed under the same conditions as Sample B with (a) 850 and (b) $1000 \mathrm{~nm}$ pore distances.

takes place at anodic potentials. The electrochemical dissolution of silicon is initiated by holes supplied from the bulk approaching the silicon-electrolyte interface which allows for nucleophilic attack of the Si atom. This attack is the rate-limiting step of the reaction and thereby the origin of pore formation. There are basically two different types of silicon etching mechanisms: one is a divalent, and the other is a tetravalent dissolution process. For anodic current densities below the critical current density $J_{\mathrm{ps}}$, the divalent dissolution process occurs and porous silicon is formed. Equation (2) is the dissolution reaction proposed for this regime.

$$
\mathrm{Si}+4 \mathrm{HF}_{2}{ }^{-}+\mathrm{h}^{+} \rightarrow \mathrm{SiF}_{6}^{2-}+2 \mathrm{HF}+\mathrm{H}_{2}+\mathrm{e}^{-}
$$

If the current density becomes larger than $J_{\mathrm{ps}}$, dissolution occurs via an intermediate anodic oxide film. Hence the reaction can be separated into electrochemical oxide formation according to eq. (3), and chemical dissolution of the oxide due to $\mathrm{HF},(\mathrm{HF})_{2}$, or $\mathrm{HF}_{2}^{-}$(i.e., electropolishing). ${ }^{18)}$

$$
\mathrm{Si}+2 \mathrm{H}_{2} \mathrm{O}+4 \mathrm{~h}^{+} \rightarrow \mathrm{SiO}_{2}+4 \mathrm{H}^{+}
$$

\subsection{The effect of external magnetic field}

The electrochemical dissolution of silicon is initiated by holes in both reactions, and thus it is critical to control the motion of holes to improve the etching properties of a silicon electrode. The external magnetic field is considered to play a significant role in this process.

Figure 7 illustrates the motion of holes generated at the pore tips by tunneling (a) without and (b) with an external magnetic field. The direction of the magnetic field is indicated by the down arrow in the figure. In the case of Fig. 7(a), holes diffuse in the pore wall regions, 
(a)

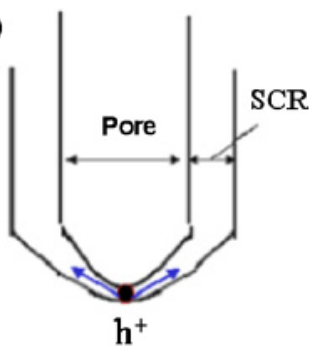

(b)

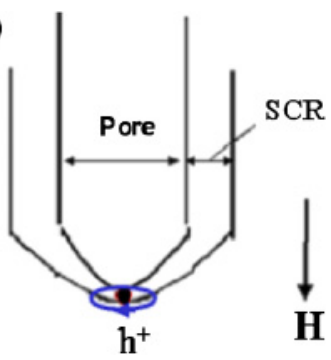

Fig. 7. (Color online) The schematic of the motion of holes generated at the pore tips by tunneling (a) without and (b) with an external magnetic field. The direction of the magnetic field is indicated by the down arrow.

which causes the undesirable etchings, such as spiking or bending of pores. On the other hand, in the case of Fig. 7(b), the motion of holes diffusing from pore tips to pore walls is restricted by the Lorentz force induced by the external magnetic field applied parallel to the direction of pore growth. Therefore, the effect of the external magnetic field is to avoid undesirable etchings, such as spiking or bending of pores which tends to occur at pore walls.

\subsection{Evidence of tetravalent dissolution process at pore walls}

The experimental results show that during the anodization process, pore tips are covered by $\mathrm{SiO}_{2}$, which is the intermediate product of tetravalent dissolution, and the oxide is formed on the pore walls as well, as indicated by the circles in Fig. 1(b). This observation indicates that almost no divalent dissolution occurs at the pore walls, which indicates that diffusion of holes to pore walls is effectively restricted by the external magnetic field. In contrast with the macropore formation processes, microporous thin films which are the products of divalent dissolution processes are not formed around pores. Therefore, the overall reactions are considered to take place via tetravalent dissolution.

\subsection{Origin of pore shapes}

As we can see in Fig. 2, sample surface geometry is not related to the pore shapes in deep regions, even immediately under the EB resist. Nevertheless, pore shapes and pore wall orientations are uniform at a depth of $10 \mu \mathrm{m}$, as indicated in Fig. 3(b). Considering that the overall reactions around pore tips mainly take place via tetravalent dissolution, the most critical issue for the origin of pore shapes lies in the orientation dependence of the critical current density for electropolishing $\left(J_{\mathrm{ps}}\right)$, which becomes maximum in (100) direction. $^{2)}$

In the direction of pore growth, the reaction occurs under electropolishing conditions because the current densities at pore tips are not limited by tunneling of charge carriers but by the reaction kinetics and ion diffusion in the solution; therefore, the current density at pore tips is likely to exceed $J_{\mathrm{ps}}$ compared with pore walls. In directions of planes, on the other hand, the reaction occurs under electropolishing conditions only in (110) direction, as indicated in Fig. 3(b), because the current density decreases from tips to pore walls and is insufficient for electropolishing in (100) direction.
This is thought to be the reason behind the uniformity of pore shapes and pore wall orientations.

\subsection{Flatness of pore walls}

Since the maximum value of $J_{\mathrm{ps}}$ is realized in (100) direction in silicon, mesoporous structures tend to grow in this direction. ${ }^{2)}$ This orientation dependence is caused by the anisotropic properties of $J_{\mathrm{ps}}$. The dissolution rate becomes maximum in (100) direction; therefore, pore tips are selectively formed in this direction. This preferential direction of mesopore growth is reduced for high doping densities and moderate to low current densities due to severe branching of pores. ${ }^{13)}$ The growth direction of a pore at high doping densities becomes dominated by the local electric field distribution at the pore tip, which depends on the position of individual dopant atoms with reference to the pore tip. Holes are attracted by such local electric field distributions which causes the degradation of pore wall quality. This has been the essential problem with existing mesopore formation techniques.

However, we have succeeded in fabricating mesopores with highly flat pore walls and a high aspect ratio of approximately 160 using magnetic field assisted anodization as shown in Fig. 5. This result, specifically the highly flat pore walls, strongly supports the discussions in earlier sections, which state that not only pore tips but pore walls are also etched under electropolishing conditions (i.e., tetravalent dissolution).

It should be noted that conditions for etching highly flat pore walls have a very narrow window. Figure 6 shows that a small change in pore distances causes a significant change in pore wall etching properties. If we compare both samples in Fig. 6, the current density during anodization is the same $\left(12 \mathrm{~mA} / \mathrm{cm}^{2}\right)$, while the pore densities differ by a factor of 1.38. On the other hand, the critical current densities differ by a factor of 1.28 at a maximum between (100) and (110) directions. ${ }^{2)}$ Therefore, the change in pore distances in Fig. 6 is considered to be sufficient to break the relationship between the critical current densities in (100) and (110) directions and the current flow at each pore tip, which makes the divalent dissolution process dominant rather than the tetravalent dissolution process. This change in distance is considered to be the cause of the spiking in Fig. 6(a).

\section{Conclusions}

We have shown the etching properties of silicon on $100-\mathrm{nm}$ scale fabricated by magnetic-field-assisted anodization. First of all, we showed that both pore tips and pore walls are covered by $\mathrm{SiO}_{2}$ during anodization, which indicates that all reactions take place mainly via tetravalent dissolution process (i.e., electropolishing). Next, we showed that the pore shapes and pore wall orientations are uniform at a depth of $10 \mu \mathrm{m}$, which is attributed to the orientation dependence of the critical current density for electropolishing in silicon substrate. Highly directional etching, $100 \mathrm{~nm}$ in diameter with an aspect ratio of 160 and with no spiking on the pore walls, is realized in the sample with highly controlled surface structrures, which supports the points of view in the discussion. These etching mechanisms enable highly controlled etching in silicon at a diameter below $100 \mathrm{~nm}$. 
1) V. Lehmann and H. Foll: J. Electrochem. Soc. 137 (1990) 653.

2) V. Lehmann: J. Electrochem. Soc. 140 (1993) 2836.

3) G. J. Parker, M. D. B. Charlton, M. E. Zoorob, J. J. Baumberg, M. C. Netti, and T. Lee: Philos. Trans. R. Soc., Ser. A 364 (2006) 189.

4) A. Birner, R. B. Wehrspohn, U. Gösele, and K. Busch: Adv. Mater. 13 (2001) 377.

5) S. Matthias, F. Muller, C. Jamois, R. B. Wehrspohn, and U. Gösele: Adv. Mater. 16 (2004) 2166.

6) V. S.-Y. Lin, K. Motesharei, K.-P. S. Dancil, M. J. Sailor, and M. R. Ghadiri: Science 278 (1997) 840.

7) S. Matthias and F. Muller: Nature 424 (2003) 53.

8) V. Lehmann and U. Gruning: Thin Solid Films 297 (1997) 13.

9) J. Schilling, J. White, A. Scherer, G. Stupian, R. Hillebrand, and U. Gösele: Appl. Phys. Lett. 86 (2005) 011101.

10) S. E. Letant, T. W. van Buuren, and L. J. Terminello: Nano Lett. 4 (2004) 1705.
11) K. Nishio, K. Yasui, F. Matsumoto, K. Kanezawa, and H. Masuda: Adv. Mater. 17 (2005) 1293

12) H. W. Lau, G. J. Parker, R. Greef, and M. Hölling: Appl. Phys. Lett. 67 (1995) 1877

13) V. Lehmann, R. Stengl, and A. Luigart: Mater. Sci. Eng. B 69-70 (2000) 11 .

14) P. C. Searson, J. M. Macaulay, and F. M. Ross: J. Appl. Phys. 72 (1992) 253

15) D. Hippo, K. Urakawa, Y. Kawata, Y. Tsuchiya, H. Mizuta, N. Koshida, and S. Oda: Jpn. J. Appl. Phys. 46 (2007) 633.

16) T. Nakagawa, H. Sugiyama, and N. Koshida: Jpn. J. Appl. Phys. 37 (1998) 7186

17) S. Matthias, F. Müller, J. Schilling, and U. Gösele: Appl. Phys. A 80 (2005) 1391

18) A. Somashekhar and S. O'Brien: J. Electrochem. Soc. 143 (1996) 2885. 